\title{
Index to Volume 28
}

A

Animals, chlorophyll breakdown, 169-170

chlorophyll biology in, 168

physiological effects of

chlorophyll catabolites, 171

chlorophylls in, 168-169

ATP synthase, 7

B

Bacteriochlorophylls (BChls), 3, 136

oceans, reservoir of photosynthetic organisms and of, 136

Bilirubin biosynthesis, 208-209

Biliverdin reductase (BVR), 208

Binary ionic porphyrin nanomaterials for

energy from sunlight, 229-230

applications of cooperative binary ionic

(CBI) porphyrin nanostructures/ nanocomposites, solar energy

CBI electrocatalysts for fuel cells, 265-266

hydrogen production using porphyrin

CBI structures, 263-265

hydrogen storage, 269-270

photovoltaics and dye-sensitized solar cells (DSSC), 266-269

solar hydrogen production, 259-263

biomimetic approach using porphyrin nanostructure, 262

carbon dioxide reduction, 271-272

exciton delocalization, 252

nanosheet solid, 255

non-linear optical materials, 270-271

optical, electronic, and catalytic properties

electronic and optoelectronic properties, 252-256

photocatalysis and self-metallization to form metal nanocomposites, 256-258

photocatalytic platinization, 257 photoconductivity of $\mathrm{Zn} / \mathrm{Sn}$ clovers, 253

synthesis and structures of CBI materials,

231

deposition of CBI materials at surfaces and interfaces, 248-249

ISA of CBI structures and characterization, 231-248

two-semiconductor photocatalytic system

for light-driven water splitting, 262

UV-visible absorption, emission, and

resonance Raman spectra, 250-252

water oxidation nanodevice, 261

Biomimetic nanodevice for artificial photosynthesis, 262

C

Carbon dioxide reduction, 271-272

Cell death mutants, 162-163

Chlorophylls (Chls), 3, 168-169

allomerization of, 138

biosynthesis

metabolic pathway of, 86

perspectives for new research objectives in, 104-108

posttranscriptional control mechanisms, 105

branch starting with protoporphyrin IX, 87 cycle, 148

oceans, reservoir of photosynthetic

organisms and of, 136

Chlorophyll (Chl) breakdown, 120-121

animals, 169-170

chlorophyll biology in, 168

chlorophyll catabolites in, physiological effects of, 171

chlorophylls in, physiological effects of, 168-169

heme oxygenase $(\mathrm{HO})$ degrades iron-pheophorbidates, 171 
path of "detoxification" of

pheophorbide $a$ (Pheo $a$ ), 169

biochemistry of Chl-breakdown in higher

plants, 146-147

colorless, fluorescent Chl-catabolites from Chl-breakdown in chloroplasts, $147-155$

peripheral modifications of primary fluorescent Chl-catabolites, 155-157

biology of Chl-breakdown in plants chlorophyll catabolic mutants in plants, 161-164

evolutionary aspects of Chl-breakdown, 166-168

subcellular localization of enzymes involved in Chl-breakdown and catabolite transport, 158-161

degraded inside chloroplast, 172 expression of Chl-catabolic genes of Arabidopsis in response to abiotic and biotic stresses, 167

heme, degraded by heme oxygenase (HO)

to biliverdin (BV), 173

humans

chlorophyll biology in, 168

physiological effects of chlorophyll catabolites in, 171

physiological effects of chlorophylls in, 168-169

linear tetrapyrroles, 137

localization and interaction of catabolic enzymes, 158-160

pheophorbide $a$ oxygenase (PAO)/ phyllobilin pathway of

Chl-breakdown, 158

photooxygenation reactions, 138

in plants, 164-166

Chl-breakdown and fruit ripening, 165-166

Chl-breakdown and leaf senescence, 164-165

Chl-breakdown and stress response, 166

evolutionary aspects of Chl-breakdown, 166-168 senescent chloroplasts of higher plants pheophorbide $a$ (Pheo $a$ ), 128

structure and chemistry of Chl-catabolites

Chl-catabolites from higher plants, 138-145

from plants and their provisional nomenclature, 121-134

structure of other photosynthetic organisms and, 134-138

tetrapyrrolic photosynthetic pigments, 168 urobilinogenoidic non-fluorescent

Chl-catabolites, 132

Chlorophyll -catabolites, structure and

chemistry of

membrane transport of Chl-catabolites,

$160-161$

partial synthesis and biologically relevant chemistry of Chl-catabolites from higher plants, 138-145

from plants and their provisional nomenclature, 121-134

non-fluorescent Chl-catabolite (NCC) and -NCC-1 "pseudo-cyclic" arrangement, 125

fluorescent Chl-catabolites from plants and their structure, 126-131

$H v$-NCC-1, 122

linear tetrapyrroles as Chl-catabolites from plants and their structure, 131-134

nonfluorescent Chl-catabolite (NCC), 122

non-fluorescent Chl-catabolite (NCC) from plants and their structure, 121-126

"rusty pigment 14," 121

structure of other photosynthetic organisms and, 134-138

Chlorophyll(ide) $a$ oxygenase, 103-104

Chlorophyll synthase, 102-103

Complementary chromatic adaptation (CCA), 189

Cooperative binary ionic (CBI) porphyrin electrocatalysts for fuel cells, 265-266

Cooperative binary ionic (CBI) porphyrin materials 
phthalocyanine-based, 245

synthesis and structures of, 231

deposition of CBI materials, 248-249

ionic self-assembly (ISA) of CBI structures, 231-248

synthesized by varying the $\mathrm{pH}$ of reaction medium, 244

Cooperative binary ionic (CBI) porphyrin nanocomposites, 245

Cooperative binary ionic (CBI) porphyrin nanomaterials, 267

non-tetrapyrrole-based, 247

Cooperative binary ionic (CBI) porphyrin nanostructures, 258, 266

Cooperative binary ionic (CBI) porphyrins rafts, 266

Co-sensitizations, 305-309

Cryptomonads, 195

Cryptophycean phycobiliproteins, 199

Cryptophyte phycobiliprotein lyases, 215

Cryptophytes. See also Cryptomonads

chromophore-binding sites of phycobiliproteins and phycobilin content, 197

cryptophycean cell, 196

phycobiliproteins in

organization, 195-196

structure, 197-199

structural models of phycoerythrin (PE) of, 198

Cyanobacteria, 189

chromophore-binding sites of phycobiliproteins and phycobilin content, 193

phycobiliproteins in

organization, 189-192

structure, 192-195

types of, 190

Cyanobacterial phycobiliprotein lyases, 211-215

crystal structure of $\mathrm{CpcS}$ from

Thermosynechococcus elongatus

BP1 (PDBID: 3BDR), 214

types and their activities, 212

Cyclopheophorbide $a$ enol (cPheo $a$-enol), 136 structural formula of Chls and of $c$ Pheo $a$-enol, 137
D

Detoxified Chl-catabolite, 137

Dicyano-dichloro-benzoquinone (DDQ), 143

Dimeric porphyrins, 302-305 molecular structures of dimeric porphyrin dyes YDD0-YDD3, 303

Dioxobilane-type non-fluorescent

Chl-catabolites (DNCC), 132, 134

structures of natural, 133

Divinyl reductase, 101-102

D protein, magnesium chelatase, 48-53

alignment of amino acid sequences of, 50

cryo-EM 3D reconstruction of complex, 62

$\mathrm{N}$-terminal AAA+ module of, 49

properties, protein sequence, and structure, $48-53$

topology of, 51

Dye-sensitized solar cells (DSSC), 229, 266-269, 280

device performance of, 281

illustration, 267

\section{$\mathbf{F}$}

Femtosecond nanocrystallography development of time-resolved, 33-34

first results of, 32-33

new method of, 27-28

on structural biology, outlook of impact of, 34-35

Fenna-Matthews-Olson (FMO) antenna protein, 9

Ferredoxin-dependent bilin reductases, 202

biosynthesis of

bilirubin, 208-209

phycocyanobilin, 204-206

phycoerythrobilin, 206-207

phycourobilin, 207-208

phytochromobilin, 204

family of, 203

Ferredoxin-dependent bilin reductases

(FDBR), 202-204, 211

FLU-like proteins (FLP), 106

Fluorescence chlorophyll catabolic mutants, 164

Fluorescent chlorophyll (Chl)-catabolite (FCC), 127-129, 141 
acid-catalyzed isomerization of epi-pFCC, 142

from Chl-breakdown in chloroplasts,

$$
\text { 147-155 }
$$

$\mathrm{Chl} \mathrm{b}$ to $\mathrm{Chl}$ a conversion, 148

magnesium dechelation and dephytylation, 149-152

pheophorbide and other green catabolites with intact Chl-macrocycle, 147-148

primary fluorescent catabolites as products of red Chl-catabolite reductase, 152-153

red Chl-catabolite from oxidation by pheophorbide oxygenase, 152-153

peripheral modifications of primary, 155 hydrolysis of methyl ester function, 156-157

$O$-malonylation reactions, 157

terminal hydroxylation of ethyl side chain at C-8, 155-156

from plants and their structure, 126-131 structures of natural, 130

Fluorescent (FLU) negative regulator, 106

Free electron laser (FEL), 15, 27, 33

\section{G}

Gerontoplasts, 147

GluTR-binding protein (GBP), 106

\section{H}

Heme oxygenases (HOs), 200-202

crystal structures of HOs, 202

genes encoding HOs, 201-202

reaction, 201

Homopolymeric protein complexes, 101

$\mathrm{H}$ subunit, magnesium chelatase, 53-58

amino acid sequences of, 54

dissimilarity between $\mathrm{H}$ sequences from various organisms, 53

protein sequence, $53-55$

structural model of ID complex, 66 structure and substrate binding, 55-58

substrate binding to, 56

Humans, chlorophyll breakdown

chlorophyll biology in, 168 physiological effects of chlorophyll catabolites in, 171

physiological effects of chlorophylls in, 168-169

Hydrogen fuel cells, 265

Hydrogen production

solar, 259-263

using porphyrin CBI structures, 263-265

by $\mathrm{Zn} / \mathrm{Sn}$ nanosheets, rate of, 264

Hydrogen storage, 269-270

Hypermodified fluorescent

chorophyll-catabolite (FCC), 172

\section{I}

Inductively coupled plasma atomic emission spectroscopy (ICP-AES) data, 244

Intensity-modulated photovoltage spectroscopy (IMVS), 308

I subunit, magnesium chelatase, 45-48 alignment of amino acid sequences from photosynthetic bacteria and higher plants, 46

cryo-EM 3D reconstruction of complex, 62

$\mathrm{N}$-terminal domain, 47

properties and amino acid sequence analysis, 45

ring structure $v s$. hexameric rings formed by other AAA+ proteins, 48

structure, 45

three-dimensional arrangement, 49

three-dimensional X-ray structure of

R. capsulatus, 47

topology of, 51

$\mathbf{L}$

LAC-series porphyrins, 297

Light-harvesting chlorophyll binding protein-like protein 3 (LIL3), 103, 107

Light harvesting complex II (LHCII), 9, 12

\section{M}

Magnesium branch of tetrapyrrole biosynthesis, $86-88$ chlorophyll(ide) $a$ oxygenase, 103-104 chlorophyll synthase, 102-103 divinyl reductase, 101-102 
magnesium chelatase, 88-95

Mg protoporphyrin IX monomethylester cyclase, 97-99

oxidative cyclization reaction, 97

oxygen-dependent cyclase reaction, 98 perspectives for new research objectives in chlorophyll biosynthesis, 104-108

protochlorophyllide oxidoreductase, 99-101

$S$-adenosyl-L-methionine (SAM) Mg protoporphyrin IX methyltransferase, 95-97

Magnesium chelatase, 42-43, 88-95 amino acid residues D207, R289, and L111 in ATP-binding site, 70 assembly of Mg chelatase complex, 91 BCHI/CHLI subunit, 89 catalytic cycle of, 75

CHLD, CHLH and CHLI subunits, 90, 92 complex, structure and dynamics of

AAA+ family, 58-60 structural comparison of representative $\mathrm{AAA}+$ domains, 59

diversity of chelatases, 44-45

GUN4 protein, 92

ID complex of magnesium chelatase in different nucleotide states, 64 integrin I domain in complex with collagen, structure of, 52 mutants, 68-72

phenotypic variants obtained from seeds of heterozygous barley, 69

reaction mechanism, 72

assembly and enzymatic mechanism, 74-78

ATP hydrolysis and need for energy, 73-74

chemical considerations, $72-73$

structure of ID complex, 60-68

dynamics of ID complex, 63-64

interaction of ID complex with $\mathrm{H}$ protein, 64-67

interactions with other proteins, 67-68

structure of motor unit of, 60-63

structure of porphyrins, as substrates in activity assays, 57 subunits

D protein, $48-53$

H subunit, 53-58

I subunit, 45-48

Metal ion-dependent adhesion site (MIDAS), 51-53

Mg-containing tetrapyrroles, 44

$\mathbf{N}$

Nanocrystalline $\mathrm{TiO}_{2}$ solar cells, 280

Nanocrystallography, 28

femtosecond development of time-resolved, 33-34 first results of, 32-33 new method of, 27-28 on structural biology, outlook of impact of, 34-35

Nanodevice, 260-261

biomimetic nanodevice for artificial photosynthesis, 262

Nanosheets, 239-244

$\mathrm{N}$-ethylmaleimide sensitive factor (NSF), 51

Nonfluorescent Chl-catabolite (NCC), 122,

124, 131, 134

chemical dihydroxylation of $C j$-NCC-1, 146

chemical oxidation of, 144

Cj-NCC-1 "pseudo-cyclic" arrangement, 125

classification of, 143

decarboxylation of So-NCC-3, 146

oxidation of, 143

pyro-NCC (PyNCC), 145

structures of natural, 123

Non-linear optical materials, 270-271

Non-tetrapyrrole-based CBI nanomaterials, 247

\section{O}

Open-chain tetrapyrroles biosynthesis, phycobiliproteins, 199-211 cryptophytes, biosynthesis of open-chain tetrapyrroles in, 209-211

ferredoxin-dependent bilin reductases, 202 bilirubin biosynthesis, 208-209 phycocyanobilin biosynthesis, 204-206 
phycoerythrobilin biosynthesis, 206-207

phycourobilin biosynthesis, 207-208

phytochromobilin biosynthesis, 204

heme oxygenases, 200-202

Oxygenation event, 3-4

Oxygen-evolving complex (OEC), 5

photosystem II, 14

with surrounding protein ligands, 15

Oxygenic photosynthesis, 18, 188

\section{$\mathbf{P}$}

PAO pathway, 146

Periplast, 196

Pheophytins (Pheins), 168-169

Phycoerythrobilin (PEB) synthase (PebS), 206

crystal structures of cyanophage PebS, 207

Pheophorbide $a$ oxygenase (PAO), 146-147

Photocatalytic platinization, 257

Photosynthesis, 3-4. See also Photosynthetic proteins, structural analysis of

biomimetic nanodevice for artificial, 262

oxygenic, 18

photosynthetic apparatus, 4-7

Photosynthetic gene cluster, 43

Photosynthetic proteins, structural analysis of evolution and relevance of photosynthesis for life on earth, 3-4

femtosecond nanocrystallography development of time-resolved, 33-34

first results of, 32-33

new method of, 27-28

on structural biology, outlook of impact of, 34-35

growth and characterization of photosystem

I (PSI) nanocrystals, 28-32

oxygen-evolving complex (OEC) with

surrounding protein ligands, 15

photosystem I (See Photosystem I (PSI))

photosystem II (See Photosystem II (PSII))

protein components of electron transport chain (ETC) and ATP synthase, 5

$\mathrm{X}$-ray free electron laser (XFEL) work on self-amplified spontaneous emission (SASE) principle, 28

Photosystem I (PSI) antenna domain of PSI, 20

antenna profile of, 23

antenna system of, 22-23

challenges of structure determination of membrane proteins, example, 26-27 cyanobacteria, 4, 16, 18, 20

data collected from nanocrystal diffraction at Linac coherent light source (LCLS), 34

"diffract before destroy" principle, 28 diversity of PS I and its peripheral antenna systems, 16-17

electron transport chain (ETC) of, 24-26

with various cofactors, 24

in higher plants, 17

Linac coherent light source (LCLS)

diffraction images at AMO

beamline, 33

lipids in, 26

membrane intrinsic subunits, 21

as model system for development of new methods for structural biology, 26-27

membrane protein complex, 27

X-ray crystallography, 26

monomer, 20

overview of main architecture of, 17-18

powder diffraction patterns, 30

proteins subunits of, 18-22

"reaction center domain" of, 18

resolved femtosecond experiment, 28-29

"salting out" of protein, 29

trimer, 19

Photosystem II (PSII)

antenna profile of, 12

antenna system of, 11-13

biological processes, 8

charge separation and electron transport chain (ETC) of, 13-14

(chlorophylls) Chls and carotenoids of, 12 cyanobacteria, 10, 12, 14

dimer from different orientations, 8

electron transport chain (ETC) in, 13

lipids in, 14-15

overview of main architecture of, 7-9

oxygen-evolving complex, 14 
proteins subunits of, 10-11

protein subunits without cofactors, 11

Photosystem I-light harvesting complex

(PSI-LHC) complex, 17

Photovoltaics, 229, 266-269

Phthalocyanines, 229

Phthalocyanine-based cooperative binary ionic

(CBI) materials, 245

Phycobilins, 193-194

Phycobiliprotein lyases, 211-215

cryptophyte phycobiliprotein lyases, 215

cyanobacterial phycobiliprotein lyases, 211-215

E/F-lyases, 211-212

identification of new, 214

S-type lyases, 213

Phycobiliproteins, 188-189

biosynthesis of, 199

biosynthetic pathway to acryloyl-bilins found in cryptophytes, 210

chromophore-binding sites of, 193

chromophoric groups of cyanobacterial and red algal, 194

in cyanobacteria

organization, 189-192

structure, 192-195

cyanophage PebS and cyanobacterial

PebA, 208

distinguishing types, 198

modification and assembly, 216-217

organisms containing, 189

pigment biosynthesis and ligation, 199

applications, 217-218

biosynthesis of open-chain tetrapyrroles, 199-211

phycobiliprotein lyases, 211-215

phycobiliprotein modification and assembly, 216-217

in rhodophyta and cryptophytes

organization, 195-196

structure, 197-199

structure of, 193

Phycobilisome (PBS), 189-190

core, allophycocyanin (APC), 190

energy transfer within PBS to chlorophyll $a$-containing PSs, 191
Phycocyanobilin (PCB), 202, 205

biosynthesis, 204-206

Pcb-antenna complex of Prochlorococcus sp., 192

Phycoerythrobilin biosynthesis, 206-207

Phycourobilin biosynthesis, 207-208

Phyllobilins, 125, 171-172

Phytochromobilin biosynthesis, 204

Phytochromobilin (PФB) synthase, 202

Phytoporphyrin (PhyP), 124

Plants

biochemistry of chlorophyll

(Chl)-breakdown in higher, 146-147

colorless, fluorescent Chl-catabolites, 147-155

peripheral modifications of primary

fluorescent Chl-catabolites, 155-157

biology of chlorophyll (Chl)-breakdown in chlorophyll catabolic mutants in plants, 161-164

enzymes involved in Chl-breakdown and catabolite transport, 158-161

evolutionary aspects of Chl-breakdown, 166-168

significance of Chl-breakdown in plants, 164-166

chlorophyll catabolic mutants in,

$$
\text { 161-164 }
$$

cell death mutants, 162-163

fluorescence mutants, 164

stay-green mutants, 161-162

Plastoquinol $\mathrm{PQH}_{2}, 6$

Polymer electrolyte membrane (PEM) fuel

cells. See Hydrogen fuel cells

Porphyrins, 229, 270

coupled with $\pi$-extended chromophore, 293-298

with different types of linkers, 281-285

four-leaf clover-like dendritic structures, 235

macrocycles, 242

with ortho-substituted long alkoxy chains, 298-302

Porphyrin cooperative binary ionic (CBI) nanomaterials, 268 
Porphyrin cooperative binary ionic (CBI) nanostructures and nanocomposites to generation, storage, and utilization of solar energy, applications of

CBI electrocatalysts for fuel cells, 265-266 hydrogen production using porphyrin CBI structures, 263-265

hydrogen storage, 269-270

photovoltaics and dye-sensitized solar cells (DSSCs), 266-269

solar hydrogen production, 259-263

Porphyrin cooperative binary ionic (CBI) structures, hydrogen production using, 263-265

Porphyrin nanofibers and nanofiber bundles, 233-234

Porphyrin nanostructures, 231, 235, 256-258 biomimetic nanodevice for artificial photosynthesis, 262

uses for, 262

Porphyrin nanotubes/nanorods, 231-233

Porphyrin-platinum nanocomposites, 260

Porphyrin-sensitized solar cells, 280-281 carboxylated porphyrin sensitizers with variety of bridging units, 283

co-sensitizations, 305-309

design of push-pull zinc porphyrins, 285-293

dimeric porphyrins, 302-305

incident photon-to-current conversion efficiency (IPCE) action spectra, 291

molecular design for push-pull zinc porphyrin (YD1), 285

molecular structures

LAC-series porphyrins, 297

LD11-LD14 porphyrins, 299

ortho-substituted push-pull zinc porphyrin (YD2-oC8) and organic dye Y123, 302

of PE1 and X-PE1 porphyrins, 292

of PE1-PE4, 283

porphyrin dyes YD11-YD13, 294

porphyrins YD14-YD17, 289

of push-pull zinc porphyrins

YD2-YD8, 287

molecular structures of ortho-substituted zinc porphyrin (YD12), 306 porphyrins

coupled with $\pi$-extended chromophore, 293-298

with different types of linkers, 281-285

with ortho-substituted long alkoxy chains, 298-302

Porphyrins YD0-YD8, 288

Porphyrins YD14-YD17, 290

Primary fluorescent chlorophyll

(Chl)-catabolite ( $p$ FCC), 127

Prochlorophyte chlorophyll (Chl)-binding protein (Pcb), 192

Protochlorophyllide oxidoreductase (POR), 99-101

crystal structure of bacterial L-subunit dimer, 100

light-dependent protochlorophyllide oxidoreductase (LPOR) protein, 101

light-independent protochlorophyllide oxidoreductase (DPOR) protein, 101

Protochlorophyllide (Pchlide) reduction triggers, 101

"Pyro"- nonfluorescent chlorophyll-catabolite (PyNCC), 145

\section{$\mathbf{R}$}

Red algae. See Rhodophyta

Red chlorophyll (Chl)-catabolite (RCC), 127, 136

electrochemical reduction of, 140

from oxidation by pheophorbide oxygenase, 152-153

partial synthesis of, 139

primary fluorescent catabolites as products of red Chl-catabolite reductase, 152-153

structural formulas of, 135

Rhodophyta, 195

chromophore-binding sites of phycobiliproteins and phycobilin content, 197

phycobiliproteins in

organization, 195-196

structure, 197-199

structural models of phycoerythrin (PE) of, 198 


\section{$\mathbf{S}$}

$S$-adenosyl-L-methionine (SAM) Mg protoporphyrin IX methyltransferase, 95-97

Second order nonlinear imaging of chiral crystals (SONICC), 30-31

Shibata shift, 101

Single-walled carbon nanotubes (SWNT), 249

Solar cells, porphyrin-sensitized, 280-281

carboxylated porphyrin sensitizers with variety of bridging units, 283

co-sensitizations, 305-309

design of push-pull zinc porphyrins, 285-293

dimeric porphyrins, 302-305

incident photon-to-current conversion efficiency (IPCE) action spectra, 291

molecular design for push-pull zinc porphyrin (YD1), 285

molecular structures

LAC-series porphyrins, 297

LD11-LD14 porphyrins, 299

ortho-substituted push-pull zinc porphyrin (YD2-oC8) and organic dye Y123, 302

of PE1 and X-PE1 porphyrins, 292

of PE1-PE4, 283

porphyrin dyes YD11-YD13, 294

porphyrins YD14-YD17, 289

of push-pull zinc porphyrins

YD2-YD8, 287

molecular structures of ortho-substituted zinc porphyrin (YD12), 306

porphyrins coupled with $\pi$-extended chromophore, 293-298

porphyrins with different types of linkers, 281-285

porphyrins with ortho-substituted long alkoxy chains, 298-302

Solar energy. See Binary ionic porphyrin nanomaterials for energy from sunlight

Solar hydrogen production, 259-263
Stay-green chlorophyll catabolic mutants, $161-162$

\section{$\mathbf{T}$}

Tetrapyrrole biosynthesis, magnesium branch of, 86-88

chlorophyll(ide) $a$ oxygenase, 103-104

chlorophyll synthase, 102-103

divinyl reductase, 101-102

magnesium chelatase, 88-95

Mg protoporphyrin IX monomethylester cyclase, 97-99

oxidative cyclization reaction, 97

oxygen-dependent cyclase reaction, 98

perspectives for new research objectives in chlorophyll biosynthesis, 104-108

posttranscriptional control mechanisms, 105

proteomic analysis, 106

protochlorophyllide oxidoreductase, 99-101

S-adenosyl-L-methionine (SAM) Mg protoporphyrin IX methyltransferase, 95-97

V

Virus-induced gene silencing (VIGS) approaches, 94

\section{W}

Walker-type NTPases, 58

Water oxidation nanodevice, 261

Y

Yellow chlorophyll (Chl)-catabolites (YCC), 131,134

air-oxidation of YCC-2, 145

natural formation of, 144

senescent leaves of Cercidiphyllum

japonicum contain, 132

$\mathbf{Z}$

$\mathrm{Zn} / \mathrm{Sn}$ porphyrin clover-like nanostructures, 251 